Check for updates

Cite this: Chem. Sci., 2019, 10, 6635

๑ All publication charges for this article have been paid for by the Royal Society of Chemistry

\section{De novo synthesis, structural assignment and biological evaluation of pseudopaline, a metallophore produced by Pseudomonas aeruginosa $\dagger$}

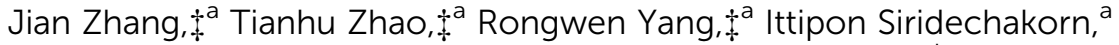
Sanshan Wang, ${ }^{a}$ Qianqian Guo, ${ }^{a}$ Yingjie Bai, ${ }^{a}$ Hong C. Shen ${ }^{b}$ and Xiaoguang Lei (iD *a

Pseudopaline is an opine carboxylate metallophore produced by Pseudomonas aeruginosa for harvesting divalent metals. However, the structure of pseudopaline is not fully elucidated. Herein, we report the first de novo total synthesis and isolation of pseudopaline, which allows unambiguous determination and confirmation of both the absolute and the relative configuration of the natural product. The synthesis highlights an efficient and stereocontrolled route using the asymmetric Tsuji-Trost reaction as the key step. The preliminary structure-activity relationship study indicated that one pseudopaline derivative shows comparable activity to pseudopaline. Moreover, a pseudopaline-fluorescein conjugate was prepared and evaluated, which confirmed that pseudopaline could be transported in the bacteria. Since the metal acquisition by $P$. aeruginosa is crucial for its ability to cause diseases, our extensive structural and functional studies of pseudopaline may pave the way for developing new therapeutic strategies such as the "Trojan horse" antibiotic conjugate against $P$. aeruginosa.
\end{abstract}

Received 22nd March 2019 Accepted 27th May 2019

DOI: $10.1039 / \mathrm{c} 9 \mathrm{sc} 01405 \mathrm{e}$

rsc.li/chemical-science transition metals ( $\mathrm{Mn}, \mathrm{Fe}, \mathrm{Co}, \mathrm{Ni}, \mathrm{Cu}$ and $\mathrm{Zn}$ ) are essential micronutrients that bacterial pathogens must acquire during the host-pathogen interactions. Bacteria have developed a metal acquisition strategy to survive under metal depleted conditions. One common strategy involves the biosynthesis of high-affinity ferric siderophores. ${ }^{6}$ Exploitation of this essential bacterial reliance on competing for iron can potentially help the development of new antibiotic therapies, such as "Trojan horse" siderophore-antibiotic conjugates. ${ }^{7}$ In fact, the most advanced drug candidate S-649266 (4) has been extensively evaluated in phase III clinical trials (Fig. 1B). ${ }^{7 \boldsymbol{h}}$

In 2016, Arnoux and co-workers described a new type of metallophore, called staphylopine (1) (Fig. 1A), that contributes to the broad-spectrum metal uptake capabilities in Staphylococcus aureus. ${ }^{8}$ Importantly, homologous biosynthetic enzymes are also encoded by other microbial pathogens, such as the Gram-negative bacteria $P$. aeruginosa. Indeed, this staphylopinelike metallophore, named pseudopaline (2), is biosynthesized from two enzymes, PaCntL and PaCntM..$^{9-11}$ Recently, a series of studies indicated that the pseudopaline operon contributes to $P$. aeruginosa virulence. The pseudopaline receptor ZrmA exhibits great relevance to lung infections in cystic fibrosis patients, and the pseudopaline operon was dramatically upregulated in the infected host sites, suggesting that the pseudopaline production is closely correlated with the $P$. aeruginosa pathogenicity. ${ }^{\mathbf{1 2 - 1 4}}$ However, pseudopaline has never been isolated from nature and the stereochemistry at the glutaric acid 
A

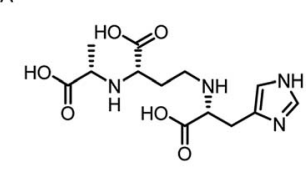

1, Staphylopine
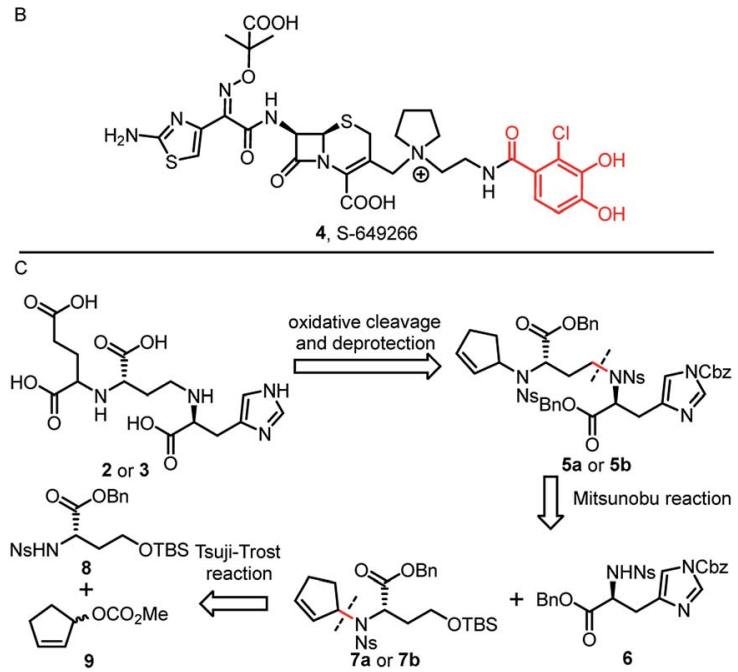

Fig. 1 (A) Chemical structures of staphylopine (1), pseudopaline (2) and epi-pseudopaline (3). (B) "Trojan horse" antibiotic-conjugate S649266 or cefiderocol (4) currently in phase III clinical trials. (C) Retrosynthetic analysis for pseudopaline.

moiety remains unclear (Fig. 1A). Therefore, total synthesis represents the practical means by which the correct structure of pseudopaline (2) can be elucidated unambiguously. ${ }^{15}$

Herein, we report the first total synthesis of pseudopaline (2) and epi-pseudopaline (3) using the asymmetric Tsuji-Trost reaction and Mitsunobu reaction as the key steps. Compared with the natural pseudopaline sample isolated from $P$. aeruginosa, we have unambiguously determined and confirmed both the absolute and the relative configuration of the natural product. Moreover, a pseudopaline-fluorescein conjugate (P-FL) 16 was prepared and evaluated. The results showed that 16 could be transported in $P$. aeruginosa. This work offers new chemical and biological insights into this crucial metallophore from Gram-negative bacteria, and a foundation for future drug discovery.

\section{Results and discussion}

Inspired by our previous synthetic studies towards carboxylate type metallophores, ${ }^{\mathbf{1 6}}$ our retrosynthetic analysis is outlined in Fig. 1C. Due to the steric hindrance of the $\mathrm{C}-\mathrm{N}$ bond at the glutaric acid moiety, no reaction was observed under the previously established Mitsunobu conditions. Therefore, we decided to invent a new approach for the synthesis of pseudopaline. The glutaric acid moiety of pseudopaline may be derived from oxidative cleavage and deprotection from 5. Cyclopentene 5 could be derived from the key intermediate 7 and L-histidine derivative 6 through the Mitsunobu reaction. We envisioned that the asymmetric Tsuji-Trost reaction might serve as a key step for the diastereoselective construction of 7 from cyclopentenyl carbonate $\mathbf{9}$ and L-homoserine derivative $\mathbf{8}$.

The asymmetric Tsuji-Trost reaction has been widely used in the synthesis of biologically active natural products. ${ }^{17 a}$ But to our knowledge, $\mathrm{N}$-cyclopentenyl asymmetric allylation using unsubstituted cyclopentene is rare. ${ }^{17 b, c}$ Our synthesis of pseudopaline commenced with deprotection of $\mathbf{1 0}^{\mathbf{1 6 b}}$ with TFA to yield free amine followed by reprotection with an $o$-nosyl group to afford the precursor 10a in moderate yield (Scheme S1 $\dagger$ ). We then screened the asymmetric Tsuji-Trost reaction conditions with 10a and 9 using $\mathrm{Pd}_{2}(\mathrm{dba})_{3} \cdot \mathrm{CHCl}_{3}$ and ligand $\mathbf{L 1}$ in THF at $0{ }^{\circ} \mathrm{C}$ for $2 \mathrm{~h}$, which mainly gave the allylation product with debenzylation of carboxylic acid (Table 1, entry 1). We hypothesized that the free hydroxyl group in 10a coordinates with $\operatorname{Pd}(0)$, which induces the hydrogenolysis of the benzyl group. $\mathrm{L}^{-}$ Homoserine 10a was then protected with TBSCl to give compound 8 (Scheme $\mathrm{S} 1 \dagger$ ). Extensive ligand examination revealed that naphtho-derived Trost ligand L2 provided an excellent diastereoselectivity with $27: 1$ d.r. (entry 3). To our delight, we could also obtain another diastereoisomer with high stereoselectivity using ent-L2 (entry 8), suggesting that there was complete reagent control. The scope of this transformation using various $o$-nosyl protected amino acid derivatives was explored (Table 2). Good to excellent yields were obtained in all

Table 1 Optimization of the asymmetric Tsuji-Trost reaction ${ }^{a}$

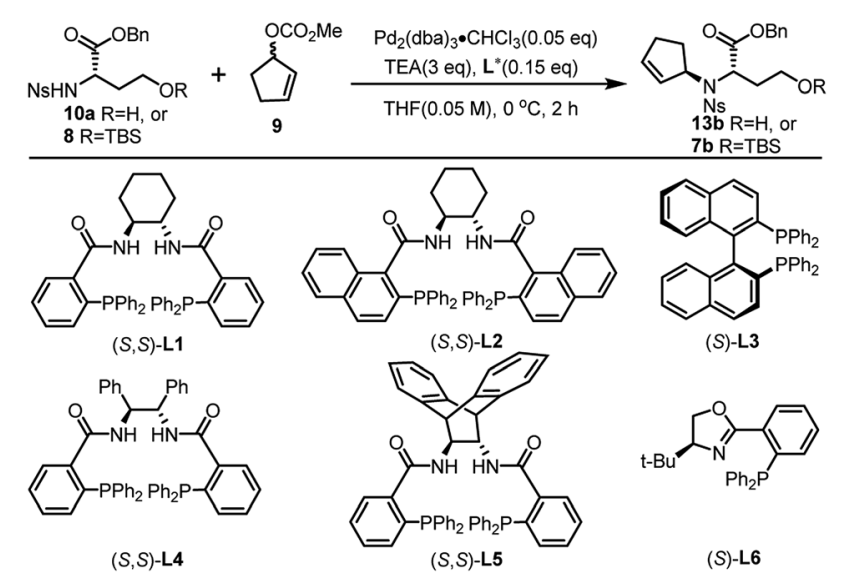

\begin{tabular}{lllll}
\hline Entry & R & Lig. & Conv. ${ }^{b}$ & d.r. $^{c}$ \\
\hline $1^{d}$ & H & L1 & Full & $2: 1$ \\
2 & TBS & L1 & Full & $2: 1$ \\
3 & TBS & L2 & Full & $27: 1$ \\
4 & TBS & L3 & Trace & - \\
5 & TBS & L4 & $92 \%$ & $3.8: 1$ \\
6 & TBS & L5 & Trace & - \\
7 & TBS & L6 & $48 \%$ & $1.9: 1$ \\
$8^{e}$ & TBS & ent -L2 & Full & $1: 27$
\end{tabular}

${ }^{a}$ Reaction conditions: $0.1 \mathrm{mmol}$ of $10 \mathrm{a}$ or $8,0.005 \mathrm{mmol}$ of $\mathrm{Pd}_{2}(\mathrm{dba})_{3} \cdot \mathrm{CHCl}_{3}, 0.015 \mathrm{mmol}$ of $\mathbf{L}, 0.3 \mathrm{mmol}$ of TEA and $\mathbf{9}$, in THF at $0{ }^{\circ} \mathrm{C}$ for $2 \mathrm{~h} .{ }^{b}$ Determined by HPLC. ${ }^{c}$ Determined by ${ }^{1} \mathrm{H}$ NMR analysis. ${ }^{d}$ Allylation product with debenzylation of carboxylic acid.

${ }^{e}$ ent-L2 provided another diastereoisomer. 
Table 2 Scope of the asymmetric Tsuji-Trost reaction ${ }^{a}$

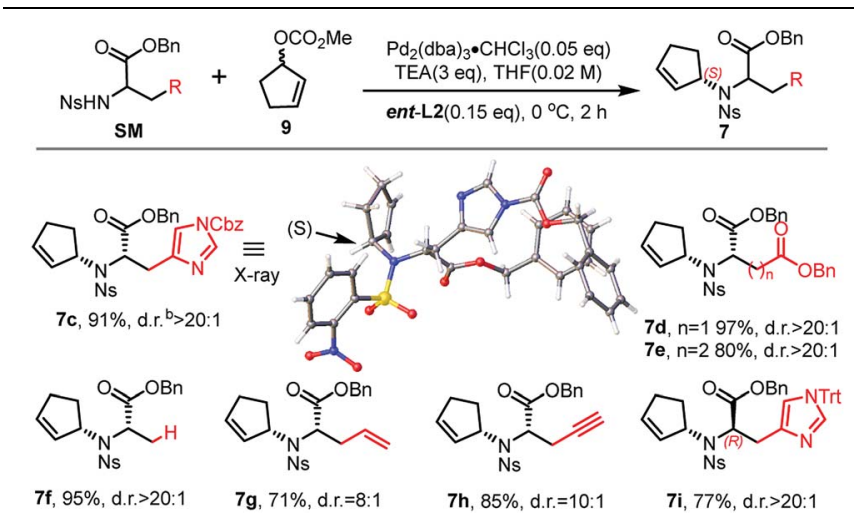

${ }^{a}$ Reaction conditions: $0.1 \mathrm{mmol}$ of $\mathrm{SM}, \quad 0.005 \mathrm{mmol}$ of $\mathrm{Pd}_{2}(\mathrm{dba})_{3} \cdot \mathrm{CHCl}_{3}, 0.015 \mathrm{mmol}$ of $\mathbf{L}, 0.3 \mathrm{mmol}$ of TEA and $\mathbf{9}$, in THF at $0{ }^{\circ} \mathrm{C}$ for 2 h. ${ }^{b}$ Determined by ${ }^{1} \mathrm{H}$ NMR analysis.

cases (absolute structure of 7c was unambiguously confirmed by X-ray crystallography and $\mathbf{7 d}$ was confirmed by chemical synthesis, see the ESI $\uparrow$ for details). These results indicate that the Tsuji-Trost conditions can be applied to synthesize various isomers of pseudopaline as well as other pseudopaline analogs for further structure-activity relationship study.

After establishing the key asymmetric Tsuji-Trost reaction conditions, we continued the total synthesis of pseudopaline and its isomer (Scheme 1). The yield of the initial route to the Tsuji-Trost precursor $\mathbf{8}$ was only moderate due to the formation of a homoserine $\gamma$-lactone byproduct under TFA or other acidic conditions (Scheme S1 $\dagger$ ). Therefore, L-homoserine 10 was first protected with TBSCl, and then the Boc group was selectively deprotected with TMSOTf followed by $o$-nosyl protection to afford compound $\mathbf{8}$ in $72 \%$ yield (Scheme 1A). In addition, we have optimized the route to generate the L-His fragment 6 . The $\mathrm{Cbz}$ protected L-His $\mathbf{1 2}^{\mathbf{1 8}}$ was produced efficiently on a large scale and purified by recrystallization. The Boc group was deprotected smoothly with TFA to yield free amine followed by protection with an $o$-nosyl group to afford the key precursor 6 in excellent yield (Scheme 1B). Applying the optimized asymmetric Tsuji-Trost reaction conditions on compounds $\mathbf{8}$ and $\mathbf{9}$, followed by deprotection of the TBS group, enabled the smooth generation of product 13a (Scheme 1C). The second $\mathrm{C}-\mathrm{N}$ bond formation between 13a and the protected L-His 13 was realized through the Mitsunobu reaction to afford the desired product 5a with the pseudopaline skeleton. However, the initial attempts for the one-step conversion to di-acid 14a with ruthenium trichloride ${ }^{19 a}$ or ozonolysis ${ }^{19 b}$ only gave complex mixtures. Gratifyingly, Lemieux-Johnson oxidation of $\mathbf{5 a}$ afforded dialdehyde, ${ }^{19 c}$ which could be further oxidized to generate di-acid 14a without purification using the Jones reagent. ${ }^{16 a}$

At this stage, deprotection of $\mathbf{1 4 a}$ would complete the synthesis of pseudopaline (2). From our previous syntheses of aspergillomarasmine $\mathrm{A}^{\mathbf{1 6 a}}$ and staphylopine, ${ }^{\mathbf{1 6 b}}$ we had utilized a global deprotection protocol. ${ }^{20}$ As expected, the benzyl and $\mathrm{Cbz}$ groups of di-acid 14a were deprotected smoothly to give diNs pseudopaline. However, the $o$-nosyl group deprotection with ethyldiisopropylamine did not go to completion even after 2 days. To our delight, the replacement of ethyldiisopropylamine with potassium carbonate could smoothly afford pure pseudopaline (2) without any racemization in 8 steps with an overall
A

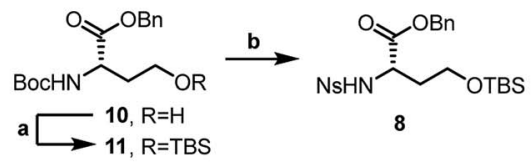

B



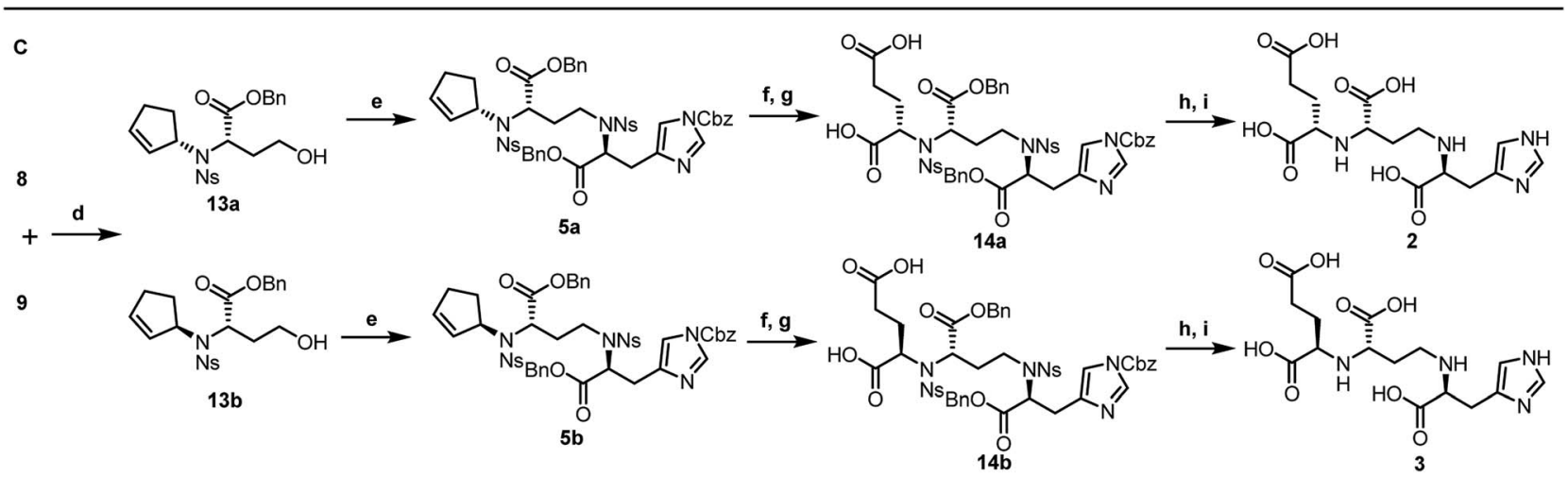

Scheme 1 (A) Synthesis of L-homoserine derivative (8). (B) Synthesis of L-histidine derivative (6). (C) Total syntheses of pseudopaline (2) and epipseudopaline (3). (a) TBSCl (1.25 eq.), imidazole (1.5 eq.), DCM (0.2 M), $0^{\circ} \mathrm{C}, 1 \mathrm{~h}, 93 \%$; (b) TMSOTf ( 8 eq.), 2,6 -lutidine (10 eq.), DCM (0.2 M), $0{ }^{\circ} \mathrm{C}$ r.t., $2 \mathrm{~h}$ then $0-\mathrm{NsCl}\left(1.2\right.$ eq.), $\mathrm{NaHCO}_{3}$ (5 eq.), EtOAc/ $\mathrm{H}_{2} \mathrm{O}(1: 1,0.2 \mathrm{M})$, r.t., $12 \mathrm{~h}, 72 \%$; (c) TFA/DCM $(1: 9,0.3 \mathrm{M}), 0{ }^{\circ} \mathrm{C}-$ r.t., $1 \mathrm{~h}$ then o- $\mathrm{NsCl}(1.1 \mathrm{eq}$.), $\mathrm{NaHCO}_{3}$ (4 eq.), EtOAc/ $\mathrm{H}_{2} \mathrm{O}(1: 1,0.2 \mathrm{M})$, r.t., $12 \mathrm{~h}, 87 \%$; (d) 9 (3eq.), $\mathrm{Pd}_{2}(\mathrm{dba})_{3}-\mathrm{CHCl}_{3}\left(0.05\right.$ eq.), ent-L2 (0.15 eq.), TEA (3 eq.), THF (0.1 M), $0{ }^{\circ} \mathrm{C}$, $2 \mathrm{~h}$; then HF.Py (4 eq.), $0{ }^{\circ} \mathrm{C}$-r.t., $10 \mathrm{~h}, 94 \%$ for $13 \mathrm{a}$; L2 afford 13b, 87\%; (e) 6 (0.7 eq.), $\mathrm{Ph}_{2} \mathrm{P}-\mathrm{Py}\left(1.5\right.$ eq.), DEAD (1.5 eq.), THF (0.1 M), $0{ }^{\circ} \mathrm{C}-$ r.t., $2 \mathrm{~h}$, $82 \%$ for $5 \mathrm{a}, 80 \%$ for $5 \mathrm{~b}$; (f) $\mathrm{OsO}_{4}\left(0.2\right.$ eq.), $\mathrm{NalO}_{4}\left(10\right.$ eq.), dioxane $/ \mathrm{H}_{2} \mathrm{O}$ (2 : 1, $\left.0.01 \mathrm{M}\right)$ 2,6-lutidine (4 eq.), r.t., 3 h; (g) Jones reagent (2.2 eq.), acetone (0.02 M), $0{ }^{\circ} \mathrm{C}$-r.t., $16 \mathrm{~h}$; $58 \%$ for $14 \mathrm{a}, 2$ steps, $55 \%$ for $14 \mathrm{~b}, 2$ steps; (h) TfOH (10 eq.), anisole (11 eq.), DCM (0.01 M), $0{ }^{\circ} \mathrm{C}, 0.5 \mathrm{~h} ; \mathrm{r.t.}, 1.5 \mathrm{~h}$; (i) $\mathrm{PhSH}$ (20 eq.), $\mathrm{K}_{2} \mathrm{CO}_{3}$ (8 eq.), DMF (0.02 M), r.t., 14 h; 74\% for 2, 2 steps, 80\% for 3, 2 steps. DEAD = Diethyl azodicarboxylate. 
$22 \%$ yield starting from the protected L-homoserine $\mathbf{1 0}$. As the stereochemistry of the glutaric acid moiety in natural pseudopaline (2) is unclear, it is essential to synthesize the $R$-glutaric acid moiety of pseudopaline for comparison. Accordingly, the chemical synthesis of epi-pseudopaline (3) was also accomplished using a previously described synthetic route (Scheme 1C). Direct comparison with natural pseudopaline is the most convincing way to elucidate the correct structure. ${ }^{21}$ However, due to the extremely high polarity of pseudopaline (5), the direct isolation turned out to be very challenging. Then we decided to in situ generate Fmoc-pseudopaline using $N$-(9-fluorenylmethoxycarbonyloxy)succinimide (Fmoc-NHS) in order to improve the detection sensitivity and decrease the polarity of pseudopaline. ${ }^{22}$ Starting from 4.2 liters of VBMM culture medium cultivated with the wild type $P$. aeruginosa PAO1, $1.1 \mathrm{mg}$ of natural Fmoc-pseudopaline was obtained (Scheme $\mathrm{S} 3 \uparrow){ }^{23}$ In addition, we have prepared the Fmoc derivative of synthetic pseudopaline for data comparison (Scheme S2 and $\mathrm{S} 4 \dagger)$. Through extensive data comparison, Fmoc-pseudopaline from synthetic and natural sources exhibited indistinguishable NMR spectra and optical rotation values. Furthermore, after mixing our synthetic Fmoc-pseudopaline with natural Fmoc-pseudopaline (in a 1:1 ratio), the ${ }^{1} \mathrm{H}$ NMR spectrum showed only one set of peaks (see the ESI $\dagger$ for details). Collectively, these results unambiguously confirmed the chemical structure of pseudopaline (2), and the experimentally determined structure matched the structures proposed in the literature. ${ }^{9}$

As the pseudopaline operon was discovered in the zincdeficient environment and subsequently was found involved in zinc uptake, ${ }^{9 a, 10,12}$ the role of pseudopaline (2) for zinc import in $P$. aeruginosa was evaluated. The intracellular zinc concentration with and without pseudopaline or epi-pseudopaline (3) was measured in a metal chelated VBMM medium (supplied with EDTA). ${ }^{10}$ The intracellular concentration of zinc significantly increased when supplied with pseudopaline (2) (Fig. 2A). The effect of pseudopaline in transporting other transition metals was also investigated. The intracellular contents of nickel, cobalt and iron significantly increased when supplied with pseudopaline (2) (Fig. 2A). These results were consistent with previous studies that this operon (PA4834-PA4837, zrmABCD) was involved in zinc, nickel, cobalt and iron uptake. $^{9 a, 10,14 a}$ No significant alterations in the intracellular contents of copper, manganese, magnesium or calcium were observed (Figure S1 $\dagger$ ). However, epi-pseudopaline showed no effect on metal import (Fig. 2A). Further experiments revealed that in the metal-rich VBMM medium (without EDTA) or nutrient-rich (LB) medium, pseudopaline (2) could not promote metal uptake (Figure S2 $\dagger$ ). The results suggest that pseudopaline (2) mediates metal uptake especially in the nutrient-depleted environment, and the $S$-configuration of pseudopaline (2) is necessary for this activity.

According to the recently reported co-crystal structure of staphylopine (staphylopine and pseudopaline share the aminobutyrate and histidine moieties while pseudopaline differs from staphylopine with the stereochemistry of its histidine moiety associated with an alpha ketoglutarate moiety instead of
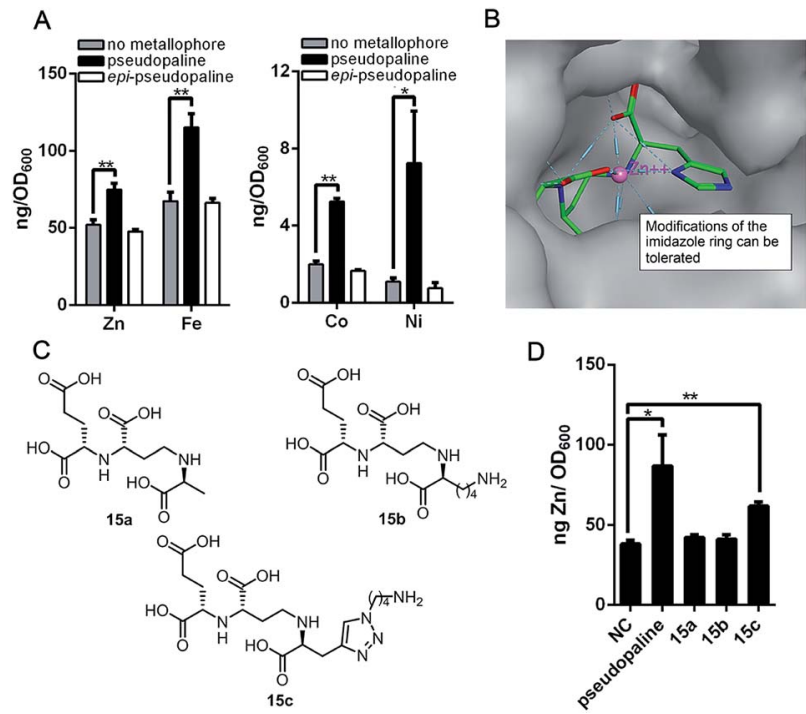

Fig. 2 The metal import by pseudopaline and epi-pseudopaline in $P$. aeruginosa in the mineral scarce medium and the design of three pseudopaline analogs $15 \mathrm{a}-\mathrm{c}$. (A) Intracellular zinc, nickel, cobalt and iron contents of wild type $P$. aeruginosa grown in VBMM containing the corresponding metal and $50 \mu \mathrm{M}$ EDTA and treated with and without $100 \mu \mathrm{M}$ pseudopaline or epi-pseudopaline measured by ICPMS from three independent trials. (B) The co-crystal structure of the CntA/staphylopine/ $/ \mathrm{n}^{2+}$ complex. ${ }^{24}$ (C) The chemical structures of the three pseudopaline analogs $15 a-c$. (D) Intracellular zinc contents of wild type $P$. aeruginosa grown in VBMM containing $10 \mu \mathrm{M}$ and $50 \mu \mathrm{M}$ EDTA and treated with and without $100 \mu \mathrm{M}$ pseudopaline, 15a, 15b or 15c, measured by ICP-MS from three independent trials. Error bars, mean $\pm \mathrm{SD} . * P<0.05$ and $* * P<0.01$

pyruvate) and its transporting protein $\mathrm{CntA},{ }^{24}$ we postulated that the imidazole ring is essential for metal chelation but at the same time could be modified without interfering with the metal-chelating and protein-binding activities. As shown in Fig. 2B, the co-crystal structure indicates that the imidazole ring forms a coordinate bond with the metal and meanwhile it is the only moiety of the natural product that points toward the outside of the protein-binding pocket. Based on this information, we rationally designed and applied our established synthetic route to prepare three pseudopaline analogs 15a-c (Fig. 2C) to examine whether the imidazole ring on pseudopaline is indispensable for metal binding and metal uptake capability (Scheme S5, $\uparrow$ the structure of $\mathbf{2 0 b}$ was confirmed by Xray crystallography). The ability of zinc transport was used to evaluate the activity of the pseudopaline analogs (Fig. 2D). Although the average intracellular zinc content of the bacteria treated with $15 \mathrm{c}$ was $28 \%$ lower than that treated with pseudopaline (2), the difference was not statistically significant $(P>$ 0.05), indicating that the change of the original imidazole ring to the substituted triazole ring could preserve the activity of zinc transport. However, the other two analogs 15a and 15b without the chelation moiety showed much reduced activities. To measure the metal binding affinity of pseudopaline (2), epipseudopaline (3) and the positive analog 15c, the stability constants for pseudopaline-metal complexes were estimated by metal competition experiments using the ESI-MS method. ${ }^{8 a, 16 b, 25}$ 
Interestingly, all the tested compounds showed strong coordination properties toward bivalent metal ions as shown by the complex dissociation constants (Table 3). Both pseudopaline (2) and epi-pseudopaline (3) show higher or comparable metal binding affinity to staphylopine (1), which suggests that the carboxyl group of glutamate side chain does not participate in the metal coordination and therefore the configuration of the glutaric acid moiety does not affect metal binding. Accordingly, we propose that the configuration of the glutaric acid moiety is important for forming the desired interactions in the proteinbinding pocket, as epi-pseudopaline cannot promote metal transport into the bacteria (Fig. 2A). To our delight, the pseudopaline analog (15c) showed comparable binding affinity to the parent natural product pseudopaline (2), together with its equivalent biological activity to pseudopaline (2), which suggests that functionalized $\mathbf{1 5 c}$ will be a promising candidate for the antibiotic conjugate.

Fluorescent siderophores have been widely used as invaluable tools to investigate the molecular mechanisms and to facilitate the diagnosis of bacterial infections. ${ }^{26}$ To confirm the potential uptake of pseudopaline itself or pseudopaline analog 15c by $P$. aeruginosa, a pseudopaline-fluorescein probe 16

Table 3 The putative metal-chelation structures and the estimated $\mathrm{p} K_{\mathrm{d}}$ values in comparison with those of staphylopine ${ }^{a}$
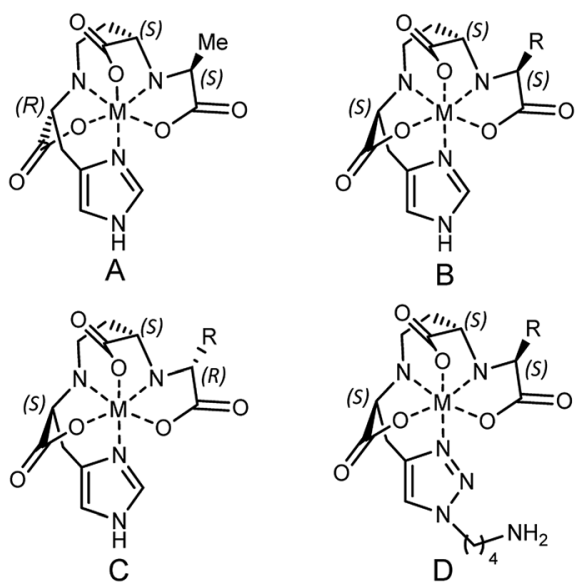

\begin{tabular}{lllll} 
& $\mathrm{p} K_{\mathrm{d}}$ & & & \\
\cline { 2 - 5 } $\begin{array}{l}\text { Metal } \\
\text { ions }\end{array}$ & Staphylopine & & & \\
\hline & Pseudopaline & epi-Pseudopaline & $\mathbf{1 5 c}$ \\
\hline $\mathrm{Cu}^{2+}$ & 19.0 & 19.4 & 19.6 & 19.3 \\
$\mathrm{Ni}^{2+}$ & 16.4 & 16.6 & 16.6 & 17.0 \\
$\mathrm{Co}^{2+}$ & 15.1 & 16.1 & 14.9 & 15.7 \\
$\mathrm{Zn}^{2+}$ & 15.0 & 15.8 & 14.7 & 15.5 \\
$\mathrm{Fe}^{2+}$ & 12.3 & 13.1 & 12.9 & 13.3 \\
$\mathrm{Mn}^{2+}$ & 9.1 & 10.4 & 9.3 & 9.7
\end{tabular}

${ }^{a}$ The putative chemical structure of the staphylopine-metal complex (A), pseudopaline-metal complex (B), epi-pseudopaline-metal complex (C) and 15c-metal complex (D). $\mathrm{R}=\left(\mathrm{CH}_{2}\right)_{2} \mathrm{COOH}$. The estimated $\mathrm{p} K_{\mathrm{d}}$ values for staphylopine (1), pseudopaline (2), epi-pseudopaline (3) and the pseudopaline analog (15c) using the competition experiments with six different metal species by ESI-MS. ${ }^{8 a} \mathrm{p} K_{\mathrm{d}}$ represents the dissociation equilibrium constant of coordination complexes.
A
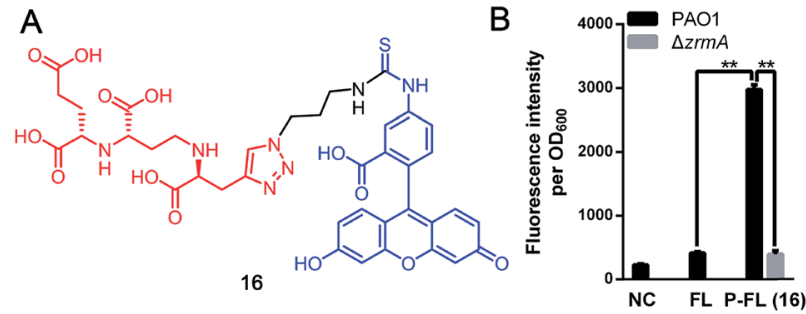

C

PA01/P-FL(16)
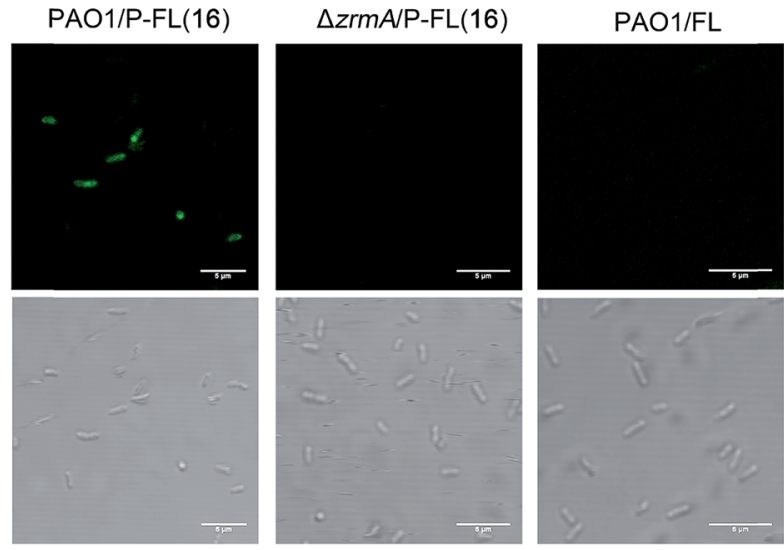

Fig. 3 Pseudopaline-fluorescein conjugate (P-FL 16) could be transported in $P$. aeruginosa. (A) The chemical structure of pseudopalinefluorescein (P-FL 16). (B) The fluorescence intensity of wild type $P$. aeruginosa $\mathrm{PAO} 1$ treated with fluorescein (5-carboxyfluorescein, FL) or pseudopaline-fluorescein (P-FL 16), and pseudopaline receptor knock-out strain $\Delta z r m A$ treated with pseudopaline-fluorescein (P-FL 16) and detected by using a microplate reader. NC, negative control, PAO1 treated without FL. (C) Fluorescence microscopy images of PAO1 and $\triangle z r m A$ treated with pseudopaline-fluorescein (P-FL 16), and PAO1 treated with fluorescein (FL). The top pictures were taken at: ex. $493 \pm 20 \mathrm{~nm} / \mathrm{em}$. $516 \pm 20 \mathrm{~nm}$. The bottom pictures were taken by light microscopy (bright phase). Scale bar: $5 \mu \mathrm{m}$. Error bars, mean \pm SD. $* * P<0.01$.

(Fig. 3A, P-FL 16) was designed and synthesized (Scheme S6†). The fluorescence intensity of $P$. aeruginosa treated with P-FL 16 in the metal-limited medium was further investigated. When the wild type $P$. aeruginosa PAO1 was incubated with P-FL 16, the fluorescence signal was clearly observed and the bacterial cell was stained (Fig. 3B, C and S3†). In contrast, when the pseudopaline outer membrane receptor knock-out strain $\Delta z r m A^{\mathbf{1 1}}$ was treated with P-FL 16, the bacteria did not exhibit a detectable fluorescence signal (Fig. 3B, C and S3 $\dagger$ ). Meanwhile, when PAO1 was incubated with the fluorescein (5-carboxyfluorescein, FL) alone under the same conditions, no significant fluorescence signal was detected either. Collectively, these data have revealed that the pseudopaline component of the probe is indispensable for the fluorescence enhancement, and illustrated that P-FL can be transported in the bacteria, and further confirmed that ZrmA is the pseudopaline receptor.

\section{Conclusions}

In summary, we have accomplished the first de novo total synthesis of pseudopaline (2), a metallophore produced by $P$. 
aeruginosa, in 8 steps from the known L-homoserine 10. The key synthetic strategy relies on the asymmetric Tsuji-Trost reaction and Mitsunobu reaction to form the challenging $\mathrm{C}-\mathrm{N}$ bonds. The total synthesis along with the natural product isolation allowed data comparison, which led to the unambiguous structural assignment of pseudopaline. The synthetic pseudopaline (2) has been shown to transport metals such as zinc, cobalt, iron, and nickel, but not copper, manganese, calcium or magnesium. The total synthesis also enabled the preparation of the inactive epi-pseudopaline (3), which demonstrated the importance of the $(S)$-configuration for metallophore function. The preliminary structure-activity relationship study indicated that pseudopaline derivative 15c shows comparable activity to pseudopaline. Moreover, a pseudopaline-fluorescein conjugate P-FL 16 was prepared and evaluated. The results showed that PFL 16 could be transported in $P$. aeruginosa. Our study demonstrates the proof-of-concept that pseudopaline could be used to develop a new "Trojan horse" antibiotic conjugate. Collectively, this work provides a solid foundation for further chemical and biological investigations to uncover the new mechanisms about how this novel metallophore transports metal ions for bacteria as well as a guide to develop new effective antibiotics against Gram-negative bacterial infections.

\section{Conflicts of interest}

There are no conflicts to declare.

\section{Acknowledgements}

We thank Dr Hiu Chun Lam (Peking University) and Dr Maarten Vercruysse (Roche) for helpful discussions as well as Prof. Changwen Jin and Prof. Hongwei Li (Peking University) for the assistance with the NMR analysis. We also thank Prof. Xiaoran He and Prof. Jiang Zhou for the HRMS-MS analysis. Dr Rongwen Yang is supported in part by the Postdoctoral Fellowship of Peking-Tsinghua Center for Life Sciences. Financial support from the NNSFC (21625201, 21661140001, 91853202 and 21521003) and National Key Research and Development Program of China (2017YFA0505200) as well as a special research grant from Roche Pharma Research \& Early Development is gratefully acknowledged.

\section{Notes and references}

1 E. D. Brown and G. D. Wright, Nature, 2016, 529, 336-343.

2 World Health Organization, WHO publishes list of bacteria for which new antibiotics are urgently needed, http:/www.who.int/ mediacentre/news/releases/2017/bacteria-antibioticsneeded/en/.

3 J. De Smet, H. Hendrix, B. G. Blasdel, K. Danis-Wlodarczyk and R. Lavigne, Nat. Rev. Microbiol., 2017, 15, 517-530.

4 H. I. Zgurskaya, C. A. López and S. Gnanakaran, ACS Infect. Dis., 2015, 1, 512-522.

5 S. Wagner, R. Sommer, S. Hinsberger, C. Lu, R. W. Hartmann, M. Empting and A. Titz, J. Med. Chem., 2016, 59, 5929-5969.
6 Z. Ma, F. E. Jacobsen and D. P. Giedroc, Chem. Rev., 2009, 109, 4644-4681.

7 For reviews on application of siderophores, see: (a) T. A. Wencewicz and M. J. Miller, Top. Med. Chem., 2017, 26, 151-184; (b) W. Neumann, A. Gulati and E. M. Nolan, Curr. Opin. Chem. Biol., 2017, 37, 10-18. For research studies on antibiotics development using siderophores, see: (c) T. Zheng, J. L. Bullock and E. M. Nolan, J. Am. Chem. Soc., 2012, 134, 18388-18400; (d) T. Zheng and E. M. Nolan, J. Am. Chem. Soc., 2014, 136, 9677-9969; (e) J. L. Madsen, T. C. Johnstone and E. M. Nolan, J. Am. Chem. Soc., 2015, 137, 9117-9127; (f) R. Liu, P. A. Miller, S. B. Vakulenko, N. K. Stewart, W. C. Boggess and M. J. Miller, J. Med. Chem., 2018, 61, 3845-3854; $(g)$ W. Neumann, M. Sassone-Corsi, M. Raffatellu and E. M. Nolan, J. Am. Chem. Soc., 2018, 140, 5193-5201. For research work on S-649266: $(h)$ N. Kohira, J. West, A. Ito, T. Ito-Horiyama, R. Nakamura, T. Sato, S. Rittenhouse, M. Tsuji and Y. Yamano, Antimicrob. Agents Chemother., 2015, 60, 729-734.

8 (a) G. Ghssein, C. Brutesco, L. Ouerdane, C. Fojcik, A. Izaute, S. Wang, C. Hajjar, R. Lobinski, D. Lemaire, P. Richaud, R. Voulhoux, A. Espaillat, F. Cava, D. Pignol, E. B. Durant and P. Arnoux, Science, 2016, 352, 1105-1109; (b) E. M. Nolan, Science, 2016, 352, 1055-1056.

9 (a) S. Lhospice, N. O. Gomez, L. Ouerdane, C. Brutesco, G. Ghssein, C. Hajjar, A. Liratni, S. Wang, P. Richaud, S. Bleves, G. Ball, E. Borezee-Durant, R. Lobinski, D. Pignol, P. Arnoux and R. Voulhoux, Sci. Rep., 2017, 7, 17132; (b) J. S. McFarlane and A. L. Lamb, Biochemistry, 2017, 56, 5967-5971.

10 M. C. Mastropasqua, M. D'Orazio, M. Cerasi, F. Pacello, A. Gismondi, A. Canini, L. Canuti, A. Consalvo, D. Ciavardelli, B. Chirullo, P. Pasquali and A. Battistoni, Mol. Microbiol., 2017, 106, 543-561.

11 J. S. McFarlane, C. L. Davis and A. L. Lamb, J. Biol. Chem., 2018, 293, 8009-8019.

12 V. G. Pederick, B. A. Eijkelkamp, S. L. Begg, M. P. Ween, L. J. McAllister, J. C. Paton and C. A. McDevitt, Sci. Rep., 2015, 5, 13139.

13 (a) W. R. Montor, J. Huang, Y. Hu, E. Hainsworth, S. Lynch, J. W. Kronish, C. L. Ordonez, T. Logvinenko, S. Lory and J. LaBaer, Infect. Immun., 2009, 77, 4877-4886; (b) C. Beckmann, M. Brittnacher, R. Ernst, N. Miller, S. I. Mayer-Hamblett and J. L. Burns, Infect. Immun., 2005, 73, 444-452; (c) J. J. Varga, M. Barbier, X. Mulet, P. Bielecki, J. A. Bartell, J. P. Owings, I. Martinez-Ramos, L. E. Hittle, M. R. Davis, F. H. Damron Jr, G. W. Liechti, J. Puchalka, V. A. dos Santos, R. K. Ernst, J. A. Papin, S. Alberti, A. Oliver and J. B. Goldberg, BMC Genomics, 2015, 16, 883.

14 (a) M. Gi, K. M. Lee, S. C. Kim, J. H. Yoon, S. S. Yoon and J. Y. Choi, Sci. Rep., 2015, 5, 14644-14657; (b) K. L. Palmer, L. M. Mashburn, P. K. Singh and M. Whiteley, J. Bacteriol., 2005, 187, 5267-5277; (c) F. H. Damron, A. G. OglesbySherrouse, A. Wilks and M. Barbier, Sci. Rep., 2016, 6, 39172; (d) P. Bielecki, J. Puchalka, M. L. Wos-Oxley, 
H. Loessner, J. Glik, M. Kawecki, M. Nowak, B. Tummler, S. Weiss and V. A. dos Santos, PLoS One, 2011, 6, e24235.

15 For review on reassignment of the natural product structure by chemical synthesis: B. K. Chhetri, S. Lavoie, A. M. Sweeney-Jones and J. Kubanek, Nat. Prod. Rep., 2018, 35, 514-531.

16 (a) D. Liao, S. Yang, J. Wang, J. Zhang, B. Hong, F. Wu and X. Lei, Angew. Chem., Int. Ed., 2016, 55, 4291-4295; Angew. Chem., 2016, 128, 4363-4367; (b) J. Zhang, S. Wang, Y. Bai, Q. Guo, J. Zhou and X. Lei, J. Org. Chem., 2017, 82, 1364313648.

17 For recent reviews on the Tsuji-Trost reaction, see: (a) B. M. Trost and M. L. Crawley, Chem. Rev., 2003, 103, 2921. For work on $\mathrm{N}$-cyclopentenyl allylation see: $(b) \mathrm{B}$. M. Trost, M. J. Krische, V. Berl and E. M. Grenzer, Org. Lett., 2002, 4, 2005-2008; (c) Z. Pakulski, O. M. Demchuk, J. Frelek, R. Luboradzki and K. M. Pietrusiewicz, Eur. J. Org. Chem., 2004, 2004, 3913-3918.

18 Q. Meng, Z. Zhu and K. Liu, Chin. J. Chem., 2005, 25, 977. (in Chinese); for the preparation of L-His 12, see the ESI. $\dagger$

19 (a) C. L. Pereira, Y.-H. Chen and F. E. McDonald, J. Am. Chem. Soc., 2009, 131, 6066-6067; (b) M. Tanasova, M. Anyika and B. Borhan, Angew. Chem. Int. Ed., 2015, 54, 4274-4278; Angew. Chem., 2015, 127, 4348-4352; (c) W. Yu, Y. Mei, Y. Kang, Z. Hua and Z. Jin, Org. Lett., 2004, 6, 3217-3219.
20 H. Yajima, N. Fujii, H. Ogawa and H. Kawatani, J. Chem. Soc., Chem. Commun., 1974, 107-108.

21 J. A. M. Mercer, C. M. Cohen, S. R. Shuken, A. M. Wagner, M. W. Smith, F. R. Moss, M. D. Smith, R. Vahala, A. GonzalezMartinez, S. G. Boxer and N. Z. Burns, J. Am. Chem. Soc., 2016, 138, 15845-15848.

22 Y.-L. Du, H.-Y. He, M. A. Higgins and K. S. Ryan, Nat. Chem. Biol., 2017, 13, 836-838.

23 A. M. King, S. A. Reid-Yu, W. Wang, D. T. King, G. De Pascale, N. C. Strynadka, T. R. Walsh, B. K. Coombes and G. D. Wright, Nature, 2014, 510, 503-506.

24 L. Song, Y. Zhang, W. Chen, T. Gu, S. Y. Zhang and Q. Ji, Proc. Natl. Acad. Sci. U. S. A., 2018, 115, 3942-3947.

25 C. K. Kempen and S. B. Broadbelt, Anal. Chem., 2000, 72, 5411-5416.

26 For reviews on application of fluorescent siderophores, see: (a) R. Nosrati, S. Dehghani, B. Karimi, M. Yousefi, S. M. Taghdisi, K. Abnous, M. Alibolandi and M. Ramezani, Biosens. Bioelectron., 2018, 117, 1-14. For research work on fluorescent siderophores, see: $(b)$ R. Nudelman, O. Ardon, Y. Hadar, Y. Chen, J. Libman and A. Shanzer, J. Med. Chem., 1998, 41, 1671-1678; (c) S. Noël, L. Guillon, I. J. Schalk and G. L. Mislin, Org. Lett., 2011, 13, 844-847; (d) P.-H. C. Chen, S. Y. Ho, P. L. Chen, T. C. Hung, A. J. Liang, T. F. Kuo, H. C. Huang and T.-S. A. Wang, ACS Chem. Biol., 2017, 12, 2720-2724. 